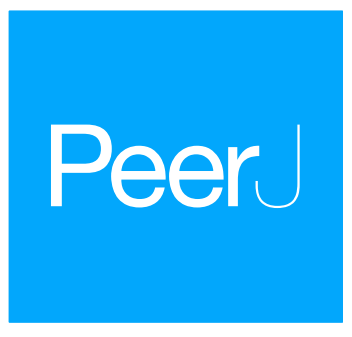

\title{
The complete mitochondrial genome sequence of Oryctes rhinoceros (Coleoptera: Scarabaeidae) based on long-read nanopore sequencing
}

\author{
Igor Filipović ${ }^{1,2}$, James P. Hereward ${ }^{1}$, Gordana Rašić ${ }^{2}$, \\ Gregor J. Devine ${ }^{2}$, Michael J. Furlong ${ }^{1}$ and Kayvan Etebari ${ }^{1}$ \\ ${ }^{1}$ School of Biological Sciences, The University of Queensland, St. Lucia, Australia \\ ${ }^{2}$ Mosquito Control Laboratory, QIMR Berghofer Medical Research Institute, Brisbane, QLD, \\ Australia
}

Submitted 10 August 2020

Accepted 21 November 2020

Published 13 January 2021

Corresponding author

Kayvan Etebari, k.etebari@uq.edu.au

Academic editor

Joseph Gillespie

Additional Information and

Declarations can be found on page 12

DOI 10.7717/peerj.10552

(c) Copyright

2021 Filipović et al.

Distributed under

Creative Commons CC-BY 4.0

\section{ABSTRACT}

Background: The coconut rhinoceros beetle (CRB, Oryctes rhinoceros) is a severe and invasive pest of coconut and other palms throughout Asia and the Pacific. The biocontrol agent, Oryctes rhinoceros nudivirus (OrNV), has successfully suppressed $O$. rhinoceros populations for decades but new CRB invasions started appearing after 2007. A single-SNP variant within the mitochondrial cox1 gene is used to distinguish the recently-invading CRB-G lineage from other haplotypes, but the lack of mitogenome sequence for this species hinders further development of a molecular toolset for biosecurity and management programmes against CRB. Here we report the complete circular sequence and annotation for CRB mitogenome, generated to support such efforts.

Methods: Sequencing data were generated using long-read Nanopore technology from genomic DNA isolated from a CRB-G female. The mitogenome was assembled with Flye v.2.5, using the short-read Illumina sequences to remove homopolymers with Pilon, and annotated with MITOS. Independently-generated transcriptome data were used to assess the $O$. rhinoceros mitogenome annotation and transcription. The aligned sequences of 13 protein-coding genes (PCGs) (with degenerate third codon position) from O. rhinoceros, 13 other Scarabaeidae taxa and two outgroup taxa were used for the phylogenetic reconstruction with the Maximum likelihood (ML) approach in IQ-TREE and Bayesian (BI) approach in MrBayes.

Results: The complete circular mitogenome of O. rhinoceros is 20,898 bp in length, with a gene content canonical for insects (13 PCGs, two rRNA genes, and 22 tRNA genes), as well as one structural variation (rearrangement of $\operatorname{trn} Q$ and $\operatorname{trnI}$ ) and a long control region (6,204 bp). Transcription was detected across all 37 genes, and interestingly, within three domains in the control region. ML and BI phylogenies had the same topology, correctly grouping O. rhinoceros with one other Dynastinae taxon, and recovering the previously reported relationship among lineages in the Scarabaeidae. In silico PCR-RFLP analysis recovered the correct fragment set that is diagnostic for the CRB-G haplogroup. These results validate the high-quality of the O. rhinoceros mitogenome sequence and annotation. 
Subjects Computational Biology, Entomology, Genomics, Molecular Biology

Keywords Mitochondrial genome, Scarabaeidae, Oryctes rhinoceros, Coconut palm pest, ONT MinION, Biosecurity and pest management

\section{INTRODUCTION}

Oryctes rhinoceros (L.) (Coleoptera: Scarabaeidae: Dynastinae), also known as the coconut rhinoceros beetle (CRB), is an important agricultural pest causing significant economic damage to coconut and other palms across Asia and South Pacific. During the 20th century, human-mediated dispersal resulted in the distribution of O. rhinoceros expanding from its native range (between Pakistan and the Philippines) throughout Oceania (Catley, 1969). After the discovery and introduction of the viral biocontrol agent Oryctes rhinoceros nudivirus (OrNV) in the 1960s, most of the CRB populations in the Pacific islands have been persistently suppressed (Huger, 2005). However, after a biocontrol campaign failed to eradicate a newly established population in Guam in 2007, new O. rhinoceros invasions were recorded in Papua New Guinea (2009), Hawaii (2013) and Solomon Islands (2015) and more recently in New Caledonia and Vanuatu (Etebari et al., 2020). Worryingly, the new invasive populations have also been difficult to control by known OrNV isolates (Marshall et al., 2017), emphasizing the importance of actively overseeing and adapting the management programmes for this important insect pest.

The expansion pathways, dynamics and hybridization of invasive insect pests and other arthropods are commonly traced through the analyses of mitochondrial sequence variation (Wang et al., 2017; Rubinoff et al., 2010; Moore et al., 2013). In the absence of a mitogenome sequence, the universal barcoding region is often amplified with degenerate primers to investigate partial sequences of $\operatorname{cox} 1$ and a limited number of other mitochondrial genes in the target species. However, analyses of such partial sequence data can fail to distinguish true mitochondrial lineages unless a sufficient number of genetic markers can be retrieved. Variation from a partial sequence of one mitochondrial gene (coxl) and one nuclear gene (cad) was not sufficient to allow confident hypotheses testing around O. rhinoceros invasion pathways (Reil, Jose \& Rubinoff, 2016), but a single diagnostic SNP within the partial cox1 gene amplicon has been used to distinguish the CRB-G haplotype from other haplotype that originally invaded the Pacific islands in the early 1900s (Marshall et al., 2017; Etebari et al., 2020). Here we report the first and complete mitogenome sequence assembly of $O$. rhinoceros, a genomic resource that will support the development of a comprehensive molecular marker toolset to help advance the biosecurity and management efforts against this resurgent pest.

The complete $O$. rhinoceros mitogenome assembly was generated using long-read Oxford Nanopore Technologies (ONT) sequencing and complemented with the short-read Illumina sequencing. The approach recovered all 37 genes (Cameron, 2014b) and a long non-coding (control) region $(6204 \mathrm{bp})$ that was not recovered in a shortread (Illumina-based) assembly, likely because it contains different putative tandem repeats. Three domains with detectable transcription within the control region and the rearrangement of two tRNA genes ( $\operatorname{trnI}$ and $\operatorname{trn} Q$ ) were also identified. The high quality of the assembly was validated through the correct placement of $O$. rhinoceros within the 
Scarabaeidae phylogeny, transcription patterns from an independently-generated transcriptome dataset, and in silico recovery of a recently reported diagnostic PCR-RFLP marker. This is the first complete mitogenome for the genus Oryctes and the subfamily Dynastinae, and among only a few for the entire scarab beetle family (Scarabaeidae).

\section{MATERIALS AND METHODS}

\section{Sample collection, DNA extraction and ONT sequencing}

An adult female O. rhinoceros was collected from a pheromone trap (Oryctalure, P046-Lure, ChemTica Internacional, S. A., Heredia Costa Rica) on Guadalcanal, Solomon Islands in January 2019 and preserved in 95\% ethanol. Mrs Helen Tsatsia (Director of Research) and members of the research team at the Ministry of Agriculture and Livestock, Honiara, Solomon Islands Government facilitated the insect collection in Solomon Islands. Initially, the mitochondrial haplotype of the specimen was determined as CRB-G (Marshall et al., 2017) via Sanger sequencing of the partial cox1 gene sequence that was amplified using the universal barcode primers LCO1490 and HCO2198 (Folmer et al., 1994). High-molecular weight DNA was extracted using a customized magnetic (SPRI) bead-based protocol. Specifically, smaller pieces of tissue from four legs and thorax $\left(50 \mathrm{~mm}^{3}\right)$ were each incubated in a $1.7 \mathrm{ml}$ eppendorf tube with $360 \mu \mathrm{L}$ ATL buffer, $40 \mu \mathrm{L}$ of proteinase K (Qiagen Blood and Tissue DNA extraction kit) for $3 \mathrm{~h}$ at RT, while rotating end-over-end at $1 \mathrm{rpm}$. A total of $400 \mu \mathrm{L}$ of AL buffer was added and the reaction was incubated for $10 \mathrm{~min}$, followed by adding $8 \mu \mathrm{L}$ of RNase A and incubation for 5 minutes. Tissue debris was spun down quickly ( $1 \mathrm{~min}$ at $16,000 \mathrm{rcf}$ ) and $600 \mu \mathrm{L}$ of homogenate was transferred to a fresh tube, where SPRI bead solution was added in 1:1 ratio and incubated for $30 \mathrm{~min}$ while rotating at end-over-end at $1 \mathrm{rpm}$. After two washes with $75 \%$ ethanol, DNA was eluted in $50 \mu \mathrm{L}$ of TE buffer. DNA quality (integrity and concentration) was assessed on the 4,200 Tapestation system (Agilent, Santa Clara, CA, USA) and with the Qubit broad-range DNA kit. To enrich for DNA $>10 \mathrm{~kb}$, size selection was done using the Circulomics Short Read Eliminator XS kit. We sequenced a total of four libraries, each prepared with $1 \mu \mathrm{g}$ of size-selected HMW DNA, following the manufacturer's guidelines for the Ligation Sequencing Kit SQK-LSK109 (Oxford Nanopore Technologies, Cambridge, UK). Sequencing was done on the MinION sequencing device with the Flow Cell model R9.4.1 (Oxford Nanopore Technologies, Cambridge, UK) and the ONT MinKNOW Software.

An Illumina sequencing library was prepared using a NebNext Ultra DNA II Kit (New England Biolabs, Ipswich, MA, USA) and was sequenced on a HiSeq X10 (150 bp paired end reads) by Novogene (Beijing, China).

\section{Mitogenome assembly, annotation and analysis}

The Guppy base caller ONT v.3.2.4 was used for high-accuracy base calling on the raw sequence data, and only high-quality sequences with a Phred score $>13$ were used for the de novo mitogenome assembly with the program Flye v.2.5 (Kolmogorov et al., 2019) in the metagenome assembly mode. The method recovered the full circular assembly and to verify its accuracy, we first mapped the original reads back to the generated mitogenome 
Table 1 Taxa with complete or partial mitogenome sequences used for the phylogenetic analyses.

\begin{tabular}{|c|c|c|c|c|c|c|}
\hline Accession & Reference & Organism & $\begin{array}{l}\text { Genome } \\
\text { type }\end{array}$ & Missing genes & $\begin{array}{l}\text { Contains } \\
\text { control region }\end{array}$ & $\begin{array}{l}\text { Sequence } \\
\text { length (bp) }\end{array}$ \\
\hline FJ859903.1 & Cameron et al. (2009) & Rhopaea magnicornis & Complete & None & Yes & 17,522 \\
\hline JX412731.1 & Timmermans et al. (2016) & Cyphonistes vallatus & Partial & $n d 1$ & No & 11,629 \\
\hline JX412734.1 & Timmermans et al. (2016) & Trox sp. & Partial & $n d 2 ; \operatorname{cox} 1$ & No & 11,622 \\
\hline JX412739.1 & Timmermans et al. (2016) & Schizonycha sp. & Partial & $n d 2$ & No & 13,542 \\
\hline JX412755.1 & Timmermans et al. (2016) & Asthenopholis sp. & Partial & $n d 2$ & No & 12,352 \\
\hline KC775706.1 & Kim et al. (2014) & Protaetia brevitarsis & Complete & None & Yes & 20,319 \\
\hline KF544959.1 & Kim \& Kim (2013) & Polyphylla laticollis mandshurica & Partial & None & No & 14,473 \\
\hline KU739455.1 & Breeschoten et al. (2016) & Eurysternus foedus & Partial & None & No & 15,366 \\
\hline KU739465.1 & Breeschoten et al. (2016) & Coprophanaeus sp. & Partial & None & No & 15,554 \\
\hline KU739469.1 & Breeschoten et al. (2016) & Bubas bubalus & Partial & None & No & 16,035 \\
\hline KU739498.1 & Breeschoten et al. (2016) & Onthophagus rhinolophus & Partial & None & No & 15,237 \\
\hline KX087316.1 & A. Hunter, 2017 (unpublished) & Melolontha hippocastani & Partial & None & No & 15,485 \\
\hline MN122896.1 & A. Margaryan, 2019 (unpublished) & Anoplotrupes stercorosus & Partial & $n d 2$ & No & 13,745 \\
\hline NC_030778.1 & Kim et al. (2016) & Osmoderma opicum & Complete & None & Yes & 15,341 \\
\hline NC_038115.1 & Yang et al. (2018) & Popillia japonica & Complete & None & Yes & 16,541 \\
\hline
\end{tabular}

assembly using Minimap2 $(\mathrm{Li}, 2018)$ with the following parameters: $-\mathrm{k} 15-$ secondary $=$ no -L -2. Second, we used BWA-MEM $(L i, 2013)$ to map short-read Illumina sequences obtained from the whole-genome sequencing of another $O$. rhinoceros female collected from the same geographic location as the specimen used for the mitogenome assembly. The read alignment analysis in Pilon (Walker et al., 2014) was used to identify inconsistencies between the draft mitogenome assembly and the aligned short Illumina reads, removing small indels that represent homopolymers (e.g., $>4$ bp single nucleotide stretches) as an inherent sequencing error of the ONT (Mikheyev \& Tin, 2014). Finally, we manually inspected if the Pilon correction occurred only in putative homopolymer regions by comparing the draft assembly with the Pilon-polished version.

The complete mitogenome sequence was initially annotated using the MITOS web server (Bernt et al., 2013), and tRNA genes and their secondary structures were cross-analysed using tRNAscan-SE v2.0 (Chan et al., 2019). To further refine the annotation and to examine mitogenome transcription, we used BWA-MEM to align Illumina reads from a transcriptome study of O. rhinoceros larvae (Shelomi, Lin \& Liu, 2019), retrieved from the NCBI (SRR9208133). Finally, we manually inspected and compared our annotation to the complete and near complete mitogenome annotations of other related taxa (Table 1) in Geneious Prime (Biomatters development team, 2020). MEGA X (Kumar et al., 2018) was used to assess the codon usage and nucleotide composition of protein-coding genes. We used Geneious Prime to test if the nucleotide sequence of the cox1 gene recovers the recently reported PCR-RFLP marker (Marshall et al., 2017). This was done by aligning the sequences of the primer pair (LCO1490 and HCO2198) to isolate the amplicon fragment and perform in silico restriction digestion with MseI restriction enzyme. The restriction digestion of the amplicon produces a set 
of fragment lengths that distinguishes CRB-G from other haplotypes (Marshall et al., 2017). The presence of tandem repeats within the control region was assessed with the Tandem Repeats Finder v.4.0.9 (Benson, 1999) using default parameters. The annotated mitogenome sequence has been deposited in GenBank under accession number MT457815.1.

\section{Phylogenetic analysis}

To ascertain if our newly sequenced $O$. rhinoceros mitogenome can be correctly placed within the Dynastinae subfamily of the Scarabaeidae family, we performed the phylogenetic analyses with 15 additional taxa for which complete or near complete mitogenome sequences could be retrieved from the NCBI. We used thirteen species from five subfamilies of the Scarabaeidae family (Dynastinae, Rutelinae, Cetoniine, Melolonthinae, Scarabaeinae) and members of two other families from Scarabaeoidea as outgroups (Trogidae, Geotrupidae) (Table 1).

Nucleotide sequences of all 13 protein-coding genes (PCGs) were first translated into amino acid sequences under the invertebrate mitochondrial genetic code and aligned using the multiple alignment in Geneious Prime. The aligned amino acid matrix was back-translated into the corresponding nucleotide matrix and the Perl script Degen v1.4 (Zwick, Regier \& Zwickl, 2012; Regier et al., 2010) was used to create the degenerated protein-coding sequences in order to reduce the bias effect of synonymous mutations on the phylogenetic analysis. These final alignments from all 13 PCGs were concatenated using Geneious Prime.

We estimated the phylogeny using two methods: the Maximum likelihood (ML) inference implemented in IQ-TREE web server (Trifinopoulos et al., 2016) and the Bayesian inference (BI) in MrBayes (Huelsenbeck \& Ronquist, 2001). For the ML analysis, the automatic and FreeRate heterogeneity options were set under optimal evolutionary models, and the branch support values were calculated using the ultrafast bootstrap (Hoang et al., 2018) and the SH-aLRT branch test approximation (Shimodaira \& Hasegawa, 1999) with 1,000 replicates. The Akaike information criterion (AIC) in ModelFinder (Kalyaanamoorthy et al., 2017) was used to select the best substitution model, and the BI phylogeny was generated with a total chain length of 1,100,000 (burn-in of 110,000 trees) and sampling every 200 cycles. The consensus trees with branch support were viewed and edited in Figtree v1.4.2 (Rambaut, 2014).

\section{RESULTS}

\section{Mitogenome assembly, organization and transcription}

The ONT long reads enabled the complete assembly of the circular mitochondrial genome for O. rhinoceros with just one MinION flow cell. This initial assembly was 20,954 bp in length and had a coverage of 3,834x. Based on the coverage ratio between the mitochondrial and draft nuclear genome assembly (unpublished data), we estimated that there were 320 copies of mitogenome for every nuclear genome copy. After including the data from three additional MinION flow cells, we produced a draft mitogenome assembly that was 21,039 bp long and had a coverage of 10,292x. Again, based on 
the coverage ratio between the mitochondrial and draft nuclear genome assembly, we estimated that there were 294 mitogenome copies per one copy of the nuclear genome in the total dataset. We then used the aligned Illumina reads to identify and correct indels, which are the most common error type produced by the ONT sequencing (Mikheyev \& Tin, 2014), resulting in the removal of $141 \mathrm{bp}$. Of those, $95 \mathrm{bp}$ were removed from the 5- to 9-mers (homopolymers). The final polished assembly was 20,898 bp in length. Our Illumina-only assembly, on the other hand, recovered a 17,665 bp mitogenome length with 3,233 bp missing from the control-region (CR).

The nucleotide composition of the complete mitogenome had high $\mathrm{A}+\mathrm{T}$ bias $(37.7 \% \mathrm{~A}$, $32.8 \% \mathrm{~T}, 19.4 \% \mathrm{C}$ and $10 \% \mathrm{G}$ ), and the long $\mathrm{CR}$ matched this genome-wide pattern (34.3\% A, 35.8\% T, 20.4\% C and 9.5\% G). The annotation revealed all 37 genes, with the rearrangement of $\operatorname{trnI}$ and $\operatorname{trn} Q$ genes, that showed the order: CR-trnQ-trnI-trnM-nad2 instead of CR-trnI-trnQ-trnM- nad2 (Table 2). CR resides within a large non-coding region (6,204 bp long) located between $r r n S$ and $\operatorname{trn} Q$, and the tandem repeats analysis revealed a complex structure of this large region with 11 putative repeats that had a consensus sequence between 7 and 410 bp repeated 2-12 times (Table 3). The length of 22 tRNAs ranged from 63 to $70 \mathrm{bp}$ (Table 2), and their predicted secondary structures exhibited a typical clover-leaf structure. The length of $r r n L$ and $r r n S$ were 1,283 bp and 783 bp, respectively (Table 2 ).

All protein coding genes (PCGs) started with a standard initiation codon (ATN), 10 of 13 PCGs terminated with the conventional stop codons (TAG or TAA), while three genes (atp6, cox3 and nad5) had an incomplete stop codon T (Table 2). In silico digestion of the cox 1 amplicon (delineated with the primer sequences from (Folmer et al., 1994)) produced the fragments $253 \mathrm{bp}$-, $138 \mathrm{bp}$-and $92 \mathrm{bp}$-long (Fig. S1) that are diagnostic for the CRB-G haplotype (Marshall et al., 2017).

The mapping of the transcriptome sequencing reads to the newly assembled mitogenome revealed that all PCGs were transcribed (mean coverage depth per base $>23000 \mathrm{X}$, Fig. 1), with cox1 and cox2 showing the highest level of expression when compared to the rRNA genes in the examined larval samples (Fig. 1). We found three domains within the large CR-containing region that also showed detectable transcription levels, with the transcript sizes of 137, 156 and 644 bp respectively. Our attempts to annotate these transcripts were not successful due to the fact that their sequences did not contain any open reading frames, nor did they have any significant BLAST hits within the NCBI's reference RNAseq or nucleotide databases.

\section{Phylogenetic analysis}

The alignment of concatenated PCG sequences of 16 species (degenerated by Degen script (Zwick, 2013)) had 1,449 parsimony-informative sites, 1,818 singleton sites and 7,823 constant sites. ModelFinder identified GTR+F+R3 as the optimal substitution model (AIC: 94067.741, AICc: 94068.053), and given the proportion of invariable sites of 0.469 and the estimated gamma shape alpha of 0.728 , we used the GTR $+\mathrm{I}+\mathrm{G}$ model as the available alternative in MrBayes. This is the most parameter-rich model that has been 


\begin{tabular}{|c|c|c|c|c|c|c|c|}
\hline $\begin{array}{l}\text { Feature } \\
\text { name }\end{array}$ & Type & $\begin{array}{l}\text { Start } \\
\text { position }\end{array}$ & $\begin{array}{l}\text { End } \\
\text { position }\end{array}$ & Length & Direction & $\begin{array}{l}\text { Start } \\
\text { codon }\end{array}$ & $\begin{array}{l}\text { Stop } \\
\text { codon }\end{array}$ \\
\hline $\operatorname{trn} Q$ & tRNA & 1 & 69 & 69 & Reverse & & \\
\hline $\operatorname{trnI}$ & tRNA & 127 & 190 & 64 & Forward & & \\
\hline $\operatorname{trn} M$ & tRNA & 195 & 263 & 69 & Forward & & \\
\hline nad2 & gene & 276 & 1,271 & 996 & Forward & ATT & TAA \\
\hline $\operatorname{trn} W$ & tRNA & 1,270 & 1,335 & 66 & Forward & & \\
\hline $\operatorname{trn} C$ & tRNA & 1,328 & 1,392 & 65 & Reverse & & \\
\hline $\operatorname{trn} Y$ & tRNA & 1,393 & 1,456 & 64 & Reverse & & \\
\hline $\operatorname{cox} 1$ & gene & 1,458 & 2,993 & 1,536 & Forward & ATC & TAA \\
\hline $\operatorname{trnL2}$ & tRNA & 2,989 & 3,054 & 66 & Forward & & \\
\hline $\cos 2$ & gene & 3,055 & 3,762 & 708 & Forward & ATA & TAA \\
\hline $\operatorname{trnK}$ & tRNA & 3,743 & 3,812 & 70 & Forward & & \\
\hline $\operatorname{trn} D$ & tRNA & 3,813 & 3,875 & 63 & Forward & & \\
\hline $\operatorname{atp} 8$ & gene & 3,876 & 4,031 & 156 & Forward & ATT & TAA \\
\hline atp6 & gene & 4,025 & 4,696 & 672 & Forward & ATG & TAT \\
\hline $\operatorname{cox} 3$ & gene & 4,695 & 5,483 & 789 & Forward & ATG & TAT \\
\hline $\operatorname{trn} G$ & tRNA & 5,482 & 5,545 & 64 & Forward & & \\
\hline nad3 & gene & 5,546 & 5,899 & 354 & Forward & ATC & TAG \\
\hline $\operatorname{trn} A$ & tRNA & 5,898 & 5,962 & 65 & Forward & & \\
\hline $\operatorname{trn} R$ & tRNA & 5,963 & 6,027 & 65 & Forward & & \\
\hline $\operatorname{trnN}$ & tRNA & 6,028 & 6,092 & 65 & Forward & & \\
\hline $\operatorname{trnS1}$ & tRNA & 6,093 & 6,159 & 67 & Forward & & \\
\hline $\operatorname{trn} E$ & tRNA & 6,161 & 6,224 & 64 & Forward & & \\
\hline $\operatorname{trnF}$ & tRNA & 6,223 & 6,288 & 66 & Reverse & & \\
\hline nad5 & gene & 6,287 & 8,005 & 1,719 & Reverse & ATT & TAT \\
\hline $\operatorname{trnH}$ & tRNA & 8,003 & 8,066 & 64 & Reverse & & \\
\hline nad4 & gene & 8,066 & 9,403 & 1,338 & Reverse & ATG & TAA \\
\hline nad4l & gene & 9,397 & 9,687 & 291 & Reverse & ATG & TAA \\
\hline $\operatorname{trn} T$ & tRNA & 9,690 & 9,754 & 65 & Forward & & \\
\hline $\operatorname{trn} P$ & tRNA & 9,755 & 9,819 & 65 & Reverse & & \\
\hline nad6 & gene & 9,821 & 10,321 & 501 & Forward & ATC & TAA \\
\hline$c o b$ & gene & 10,321 & 11,463 & 1,143 & Forward & ATG & TAG \\
\hline $\operatorname{trn} S 2$ & tRNA & 11,462 & 11,527 & 66 & Forward & & \\
\hline nad1 & gene & 11,547 & 12,497 & 951 & Reverse & ATT & TAA \\
\hline $\operatorname{trnL1}$ & tRNA & 12,499 & 12,561 & 63 & Reverse & & \\
\hline$r r n L$ & rRNA & 12,559 & 13,841 & 1,283 & Reverse & & \\
\hline $\operatorname{trn} V$ & tRNA & 13,843 & 13,912 & 70 & Reverse & & \\
\hline$r r n S$ & rRNA & 13,912 & 14,694 & 783 & Reverse & & \\
\hline Control region & misc_feature & 14,695 & 20,898 & 6,204 & None & & \\
\hline Expressed region 1 & misc_RNA & 15,117 & 15,253 & 137 & Reverse & & \\
\hline Expressed region 2 & misc_RNA & 15,321 & 15,476 & 156 & Reverse & & \\
\hline Expressed region 3 & misc_RNA & 17,744 & 18,387 & 644 & Reverse & & \\
\hline
\end{tabular}


Table 3 Characteristics of the putative tandem repeats in the control region of the Oryctes rhinoceros mitogenome.

\begin{tabular}{|c|c|c|c|c|c|c|c|c|c|c|c|}
\hline Indices & $\begin{array}{l}\text { Period } \\
\text { Size }\end{array}$ & $\begin{array}{l}\text { Copy } \\
\text { Number }\end{array}$ & $\begin{array}{l}\text { Consensus } \\
\text { Size }\end{array}$ & $\begin{array}{l}\text { Percent } \\
\text { Matches }\end{array}$ & $\begin{array}{l}\text { Percent } \\
\text { Indels }\end{array}$ & Score & $\mathbf{A}$ & $\mathrm{C}$ & G & $\mathbf{T}$ & $\begin{array}{l}\text { Entropy } \\
(0-2)\end{array}$ \\
\hline $14,764-14,795$ & 16 & 2 & 16 & 93 & 0 & 55 & 53 & 0 & 0 & 46 & 1 \\
\hline $14,757-14,797$ & 7 & 6 & 7 & 78 & 16 & 50 & 48 & 0 & 0 & 51 & 1 \\
\hline $14,818-14,859$ & 15 & 3 & 14 & 86 & 10 & 59 & 38 & 0 & 0 & 61 & 0.96 \\
\hline $15,012-15,440$ & 133 & 3.2 & 133 & 97 & 1 & 824 & 52 & 15 & 6 & 25 & 1.65 \\
\hline $15,447-17,166$ & 285 & 6 & 285 & 98 & 0 & 3,336 & 24 & 29 & 13 & 33 & 1.93 \\
\hline $16,955-17,949$ & 206 & 4.9 & 205 & 98 & 1 & 1,933 & 24 & 29 & 12 & 33 & 1.92 \\
\hline $16,955-17,949$ & 410 & 2.4 & 409 & 98 & 1 & 1,938 & 24 & 29 & 12 & 33 & 1.92 \\
\hline $18,024-18,419$ & 110 & 3.6 & 110 & 96 & 1 & 724 & 41 & 24 & 8 & 25 & 1.83 \\
\hline $18,656-18,707$ & 22 & 2.3 & 23 & 81 & 15 & 65 & 51 & 1 & 3 & 42 & 1.31 \\
\hline $19,664-20,895$ & 102 & 12 & 102 & 98 & 0 & 2,351 & 36 & 11 & 7 & 44 & 1.69 \\
\hline $19,664-20,895$ & 205 & 6 & 204 & 98 & 0 & 2,369 & 36 & 11 & 7 & 44 & 1.69 \\
\hline
\end{tabular}

shown to yield highly robust tree topologies for long alignments ( $>1,250 \mathrm{bp})$ in the smaller sample sizes (<17 taxa), irrespective of the model selection results (Abadi et al., 2019).

The topology of the maximum likelihood (IQ-tree) phylogeny was identical to the Bayesian (MrBayes) phylogeny, with O. rhinoceros being grouped with another member of the Dynastinae subfamily (Cyphonistes vallatus) with high confidence (100\% SH-aLRT support, $100 \%$ ultrafast bootstrap support in ML, posterior probability $100 \%$ in $\mathrm{BI}$ ). Dynastinae and Rutelinae were inferred as sister clades, that together with Cetoniine and Melolonthinae formed a basal split between phytophagus and coprophagus scarab beetles (Scarabaeinae) (Fig. 2).

\section{DISCUSSION}

The complete circular mitogenome sequence of $O$. rhinoceros is the only complete mitogenome assembly and annotation for the Dynastinae subfamily, and among only a few complete mitogenomes for the scarab beetles. Its size (20,898 bp) is among the largest reported in Coleoptera, and is similar in size to another scarab beetle, Protaetia brevitarsis (20,319 bp) (Kim et al., 2014). Mitogenome size is driven by the large non-coding control region (CR) that is 6,204 bp-and 5,654 bp-long in O. rhinoceros and P. brevitarsis, respectively. In Popillia mutans, another scarab beetle with a complete mitogenome sequence, the reported length of this region is only 1,497 bp (Song \& Zhang, 2018).

Variation in the size and nucleotide composition of the mitochondrial CR is not unusual in insects (Zhang \& Hewitt, 1997), however, there could also be technical reasons for some size discrepancies among taxa. Given that we recovered 17,665 bp in our Illumina-only assembly (3,233 bp were missing from the CR), it is possible that many other beetles have larger mitogenomes, but the repetitive content of CR presents a challenge for the most commonly used approaches such as Sanger or short-read NGS sequencing of long-PCR amplicons (Cameron, 2014a). While the CR size can be estimated from the size of long-PCR amplicons, its Sanger sequencing by primer walking is often impossible because it is difficult to design useful primers where GC content is insufficient 


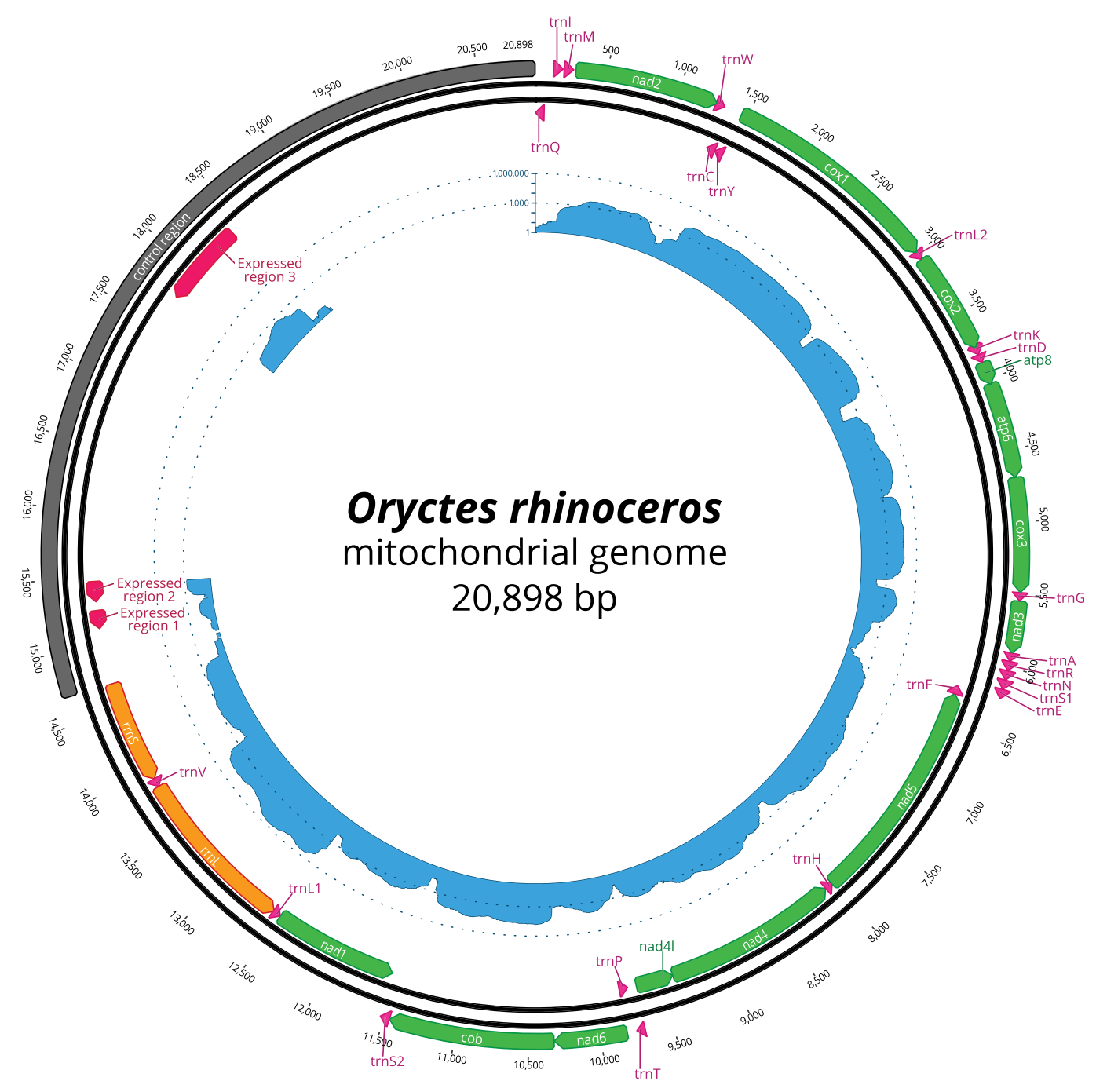

Figure 1 Circular representation of complete $O$. rhinoceros mitochondrial genome. The position and orientation of 13 PCG genes (green), 22 trna genes (pink), two rRNA genes (orange), control region (grey) with three expressed domains (red). The inner circle displays transcriptome read depth (blue) on a logarithmic scale. Full-size 0 DOI: 10.7717/peerj.10552/fig-1

and homopolymers and tandem repeats are abundant (Cameron et al., 2012; Cameron, 2014a). Long repetitive regions also complicate the assembly process with the short-read NGS sequences (either from the long-PCR amplicons or total DNA extractions) because they are often algorithmically collapsed due to their similarity. For example, three out of five scarab mitogenome assemblies recently generated with the short-read technology are incomplete and lack the control region and adjacent genes (Song \& Zhang, 2018). The use of RNA-seq transcriptome data is the least likely to recover the CR, given that this part of the mitogenome generally does not transcribe (or has few short domains of transcription, as we describe in O. rhinoceros).

Our approach included the long-read (ONT) sequencing of the libraries that were prepared with non-PCR-amplified DNA, resulting in a fully closed circular assembly with thousands of reads spanning the entire length of the control region. The superiority of 


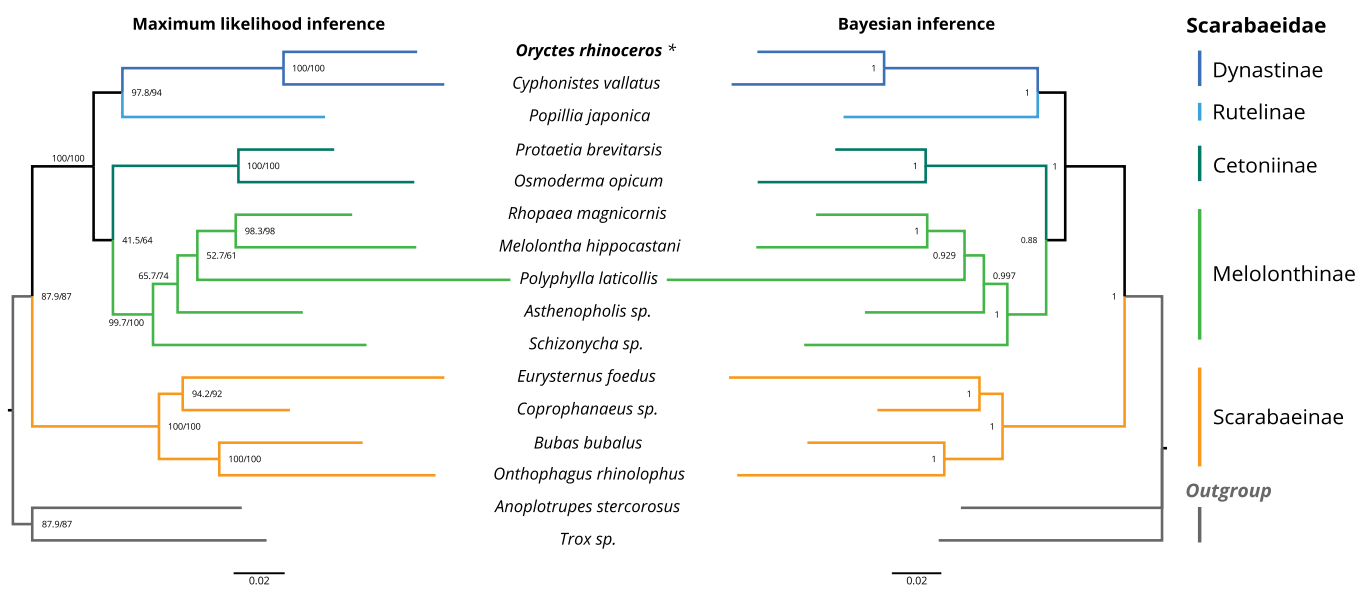

Figure 2 Maximum likelihood (ML) and Bayesian (BI) consensus tree inferred from the PCG dataset using IQ-TREE (ML) and MrBayes (BI). Branch support values for the ML consensus tree are presented near each node as SH-aLRT support (\%)/ultrafast bootstrap support (\%), and branch lengths were optimized by maximum likelihood on original alignment. Branch support values for the BI consensus tree are posterior probabilities (0-1).The colored lines correspond to Scarabaeidae subfamilies.

Full-size $\underset{0}{0}$ DOI: 10.7717/peerj.10552/fig-2

long-read sequencing technologies to capture the long repeated, AT-rich sequences has led to the discovery of remarkable interspecific variation in the length of the intergenic repeat regions in the mitogenomes of seed beetles (Chrysomelidae), that can range between 0.1 and $10.5 \mathrm{kbp}$ (Sayadi et al., 2017). However, for taxa where CR is short (e.g., Lepidoptera (Zhang \& Hewitt, 1997)), the long-read technology is not necessary for obtaining the complete mitogenome assemblies.

Unambiguous detection of gene rearrangements and other structural changes is another benefit of the long-read sequencing. In the O. rhinoceros mitogenome, $\operatorname{trn} Q$ gene precedes $\operatorname{trnI}$ gene, and this rearrangement is supported with thousands of long reads spanning this region. The rearranged position of $\operatorname{trnI}$ and $\operatorname{trn} Q$ genes is found in almost all species of Hymenoptera (Dowton et al., 2009), and was also reported in flatbugs (Hemiptera, Aradidae) (Song et al., 2016). A number of other rearrangements in tRNA genes have been reported in Lepidoptera and Neuroptera (Cao et al., 2012; Cameron et al., 2009), and because they all occurred between the CR and coxl, it has been hypothesized that this might be a 'hotspot' region for such changes (Dowton et al., 2009).

It is also important to note the higher basecalling error rate of ONT when compared to the short-read NGS or Sanger data. Using the aligned short-read sequences (Illumina, San Diego, CA, USA), we identified 141 erroneous additions of 1 or 2 nucleotides, and $67 \%$ of those were found in 5-to 9-bp homopolymers. This occurred despite achieving the depth of thousands of reads per each nucleotide position $(>10 \mathrm{k} \times$ median depth), indicating systematic errors during the basecalling process that result in erroneous consensus sequence (Wick, Judd \& Holt, 2019), and this cannot be circumvented with high sequencing depth alone. When short-read data are not available for polishing, we suggest to carefully manually inspect the alignment between the draft genome and other 
high-quality assemblies from the related taxa in order to remove most (if not all) errors in the consensus sequence.

The nucleotide composition of the final (polished) O. rhinoceros mitogenome sequence had high $\mathrm{A}+\mathrm{T}$ bias $(37.7 \% \mathrm{~A}, 32.8 \% \mathrm{~T}, 19.4 \% \mathrm{C}$ and $10 \% \mathrm{G})$, which is highly concordant with other scarab beetle species (Song \& Zhang, 2018), and the long CR matched this genome-wide pattern (34.3\% A, 35.8\% T, 20.4\% C and 9.5\% G). All PCGs start with a standard ATN codon, including cox1 that generally seems to be a hot-spot for non-canonical start codon in annotations of invertebrate genomes (Donath et al., 2019). Among 15 other Scarabaeoidea we examined (Table 1), 14 had cox1 in their mitogenome assembly, nine of which had a standard ATN codon while 5 taxa did not have a defined start codon, resulting in a different length of this gene. A more comprehensive analysis (with an extended taxon sampling) is needed to reliably quantify the extent of coxl start-codon variation in this insect group. We also found evidence of some transcriptional activity within the control region of the O. rhinoceros mitogenome, but to fully characterize this pattern, more transcriptome data (from different tissues, life stages etc.) would need to be tested. Transcriptional activity within the intergenic repeat regions has been detected in mitogenomes of seed beetles (Sayadi et al., 2017), suggesting that 'mitochondrial dark matter' could be a source of non-coding RNAs in insects.

We used phylogenetic analysis to assess the quality of the O. rhinoceros mitogenome assembly, expecting to recover its grouping with one other member of the Dynastinae subfamily. This grouping was indeed highly supported in both ML and BI phylogeny (Fig. 2). Identical topology of the ML and BI trees showed Dynastinae and Rutelinae as sister clades, that formed a basal split between phytophagous and coprophagous scarab beetles with Cetoniine and Melolonthinae (Fig. 2). It is worth noting that the intra-family phylogeny of this group is inconsistent depending on the type of the sequence data (mitogenome vs. mito+nuclear genes) used for the inference. While Melolonthinae have been reported as paraphyletic and Cetoniinae as more closely related to Dynastinae and Rutelinae, the sister-group relationship between Dynastinae and Rutelinae was recovered in all previous studies of this group (Ahrens, Schwarzer \& Vogler, 2014; Gunter et al., 2016; Song \& Zhang, 2018). Further support for the high quality of the $O$. rhinoceros mitogenome sequence and annotation was our in silico recovery of the correct PCR-RFLP marker set that is diagnostic for the invasive CRB-G haplotype (Marshall et al., 2017).

\section{CONCLUSIONS}

We report the circularized complete mitochondrial genome assembly and annotation for O. rhinoceros, the major insect pest of coconut and oil palms. The long-read ONT sequencing allowed us to identify structural variation ( $\operatorname{trnI-trnQ}$ rearrangement) and span the assembly across the entire 6,203 bp-long control region that contains tandem repeats and regions of transcriptional activity. This high-quality genomic resource facilitates future development of a molecular marker toolset to help with the biosecurity and management efforts against this resurgent pest. As the first complete mitogenome for the 
genus Oryctes and the subfamily Dynastinae, and among a few for the entire scarab beetle family (Scarabaeidae), it will contribute to the resolution of higher-level taxonomy and phylogeny of phytophagous scarab beetles that remain understudied despite containing many agricultural pests.

\section{ACKNOWLEDGEMENTS}

We would like to thank two anonymous reviewers for improving our manuscript through their insightful comments and suggestions.

\section{ADDITIONAL INFORMATION AND DECLARATIONS}

\section{Funding}

This project was supported by the Australian Centre for International Agricultural Research funding (HORT/2016/185), the University of Queensland (UQECR2057321) and by core funds from the Mosquito Control Laboratory at QIMR Berghofer MRI.

The funders had no role in study design, data collection and analysis, decision to publish, or preparation of the manuscript.

\section{Grant Disclosures}

The following grant information was disclosed by the authors:

Australian Centre for International Agricultural Research: HORT/2016/185.

University of Queensland: UQECR2057321.

Mosquito Control Laboratory at QIMR Berghofer MRI.

\section{Competing Interests}

The authors declare that they have no competing interests.

\section{Author Contributions}

- Igor Filipović: Methodology, Investigation, Data curation, Visualization, Writing Original draft preparation and manuscript finalization.

- James P. Hereward: Data curation, Writing - Reviewing and Editing.

- Gordana Rašić: Methodology, Investigation, Resources, Writing - Reviewing and Editing.

- Gregor J. Devine: Resources, Supervision, Writing - Reviewing and Editing.

- Michael J. Furlong: Funding acquisition, Project administration, Supervision, Writing Reviewing and Editing.

- Kayvan Etebari: Conceptualization, Investigation, Resources, Funding acquisition, Supervision, Writing - Reviewing and Editing and manuscript finalization.

\section{Field Study Permissions}

The following information was supplied relating to field study approvals (i.e., approving body and any reference numbers):

Mrs Helen Tsatsia (Director of Research) and members of the research team at the Ministry of Agriculture and Livestock, Honiara, Solomon Islands Government facilitated the insect collection in Solomon Islands. 


\section{Data Availability}

The following information was supplied regarding data availability:

The assembled data is available at NCBI: MT457815.1.

\section{Supplemental Information}

Supplemental information for this article can be found online at http://dx.doi.org/10.7717/ peerj.10552\#supplemental-information.

\section{REFERENCES}

Abadi S, Azouri D, Pupko T, Mayrose I. 2019. Model selection may ot be a mandatory step for phylogeny reconstruction. Nature Communications 10(1):368

DOI 10.1038/s41467-019-08822-w.

Ahrens D, Schwarzer J, Vogler AP. 2014. The evolution of scarab beetles tracks the sequential rise of angiosperms and mammals. Proceedings: Biological Sciences/The Royal Society 281(1791):20141470.

Benson G. 1999. Tandem repeats finder: a program to analyze DNA sequences. Nucleic Acids Research 27(2):573-580 DOI 10.1093/nar/27.2.573.

Bernt M, Donath A, Jühling F, Externbrink F, Florentz C, Fritzsch G, Pütz J, Middendorf M, Stadler PF. 2013. MITOS: improved de novo metazoan mitochondrial genome annotation. Molecular Phylogenetics and Evolution 69(2):313-319 DOI 10.1016/j.ympev.2012.08.023.

Biomatters development team. 2020. Geneious Prime ${ }^{\circledR}$. version 2020.0.4. Available at https://www.geneious.com/.

Breeschoten T, Doorenweerd C, Tarasov S, Vogler AP. 2016. Phylogenetics and biogeography of the dung beetle genus onthophagus inferred from mitochondrial genomes.

Molecular Phylogenetics and Evolution 105:86-95 DOI 10.1016/j.ympev.2016.08.016.

Cameron SL. 2014a. How to sequence and annotate insect mitochondrial genomes for systematic and comparative genomics research. Systematic Entomology 39(3):400-411

DOI 10.1111/syen.12071.

Cameron SL. 2014b. Insect mitochondrial genomics: implications for evolution and phylogeny. Annual Review of Entomology 59(1):95-117 DOI 10.1146/annurev-ento-011613-162007.

Cameron SL, Lo N, Bourguignon T, Svenson GJ, Evans TA. 2012. A mitochondrial genome phylogeny of termites (Blattodea: Termitoidae): robust support for interfamilial relationships and molecular synapomorphies define major clades. Molecular Phylogenetics and Evolution 65(1):163-173 DOI 10.1016/j.ympev.2012.05.034.

Cameron SL, Sullivan J, Song H, Miller KB, Whiting MF. 2009. A mitochondrial genome phylogeny of the neuropterida (lace-Wings, Alderflies and Snakeflies) and their relationship to the other holometabolous insect orders. Zoologica Scripta 38(6):575-590 DOI 10.1111/j.1463-6409.2009.00392.x.

Cao Y-Q, Ma C, Chen J-Y, Yang D-R. 2012. The complete mitochondrial genomes of two ghost moths, Thitarodes Renzhiensis and Thitarodes Yunnanensis: the ancestral gene arrangement in Lepidoptera. BMC Genomics 13:276 DOI 10.1186/1471-2164-13-276.

Catley A. 2009. The coconut rhinoceros beetle Oryctes Rhinoceros (L) (Coleoptera: Scarabaeidae: Dynastinae). PANS Pest Articles \& News Summaries 15(1):18-30 DOI 10.1080/04345546909415075.

Chan PP, Lin BY, Mak AJ, Lowe TM. 2019. tRNAscan-SE 2.0: improved detection and functional classification of transfer RNA genes. bioRxiv 7:116 DOI 10.1101/614032. 
Donath A, Frank J, Marwa A-A, Stephan HB, Franziska R, Peter FS, Martin M, Matthias B. 2019. Improved annotation of protein-coding genes boundaries in metazoan mitochondrial genomes. Nucleic Acids Research 47(20):10543-10552 DOI 10.1093/nar/gkz833.

Dowton M, Cameron SL, Dowavic JI, Austin AD, Whiting MF. 2009. Characterization of 67 mitochondrial tRNA gene rearrangements in the hymenoptera suggests that mitochondrial tRNA gene position is selectively neutral. Molecular Biology and Evolution 26(7):1607-1617 DOI 10.1093/molbev/msp072.

Etebari K, Hereward J, Sailo A, Ahoafi EM, Tautua R, Tsatsia H, Jackson GV, Furlong MJ. 2020. Genetic structure of the coconut rhinoceros beetle (Oryctes Rhinoceros) population and the incidence of its biocontrol agent (Oryctes Rhinoceros Nudivirus) in the South Pacific Islands. bioRxiv 6(3):27 DOI 10.1101/2020.07.30.229872.

Folmer O, Black M, Hoeh W, Lutz R, Vrijenhoek R. 1994. DNA primers for amplification of mitochondrial cytochrome c oxidase subunit I from diverse metazoan invertebrates. Molecular Marine Biology and Biotechnology 3(5):294-299.

Gunter NL, Weir TA, Slipinksi A, Bocak L, Cameron SL. 2016. If dung beetles (Scarabaeidae: Scarabaeinae) arose in association with dinosaurs, did they also suffer a mass co-extinction at the K-Pg boundary? PLOS ONE 11(5):e0153570 DOI 10.1371/journal.pone.0153570.

Hoang DT, Chernomor O, Von Haeseler A, Minh BQ, Vinh LS. 2018. UFBoot2: improving the ultrafast bootstrap approximation. Molecular Biology and Evolution 35(2):518-522 DOI 10.1093/molbev/msx281.

Huelsenbeck JP, Ronquist F. 2001. MRBAYES: Bayesian inference of phylogenetic trees. Bioinformatics 17(8):754-755 DOI 10.1093/bioinformatics/17.8.754.

Huger AM. 2005. The oryctes virus: its detection, identification, and implementation in biological control of the coconut palm rhinoceros beetle, Oryctes Rhinoceros (Coleoptera: Scarabaeidae). Journal of Invertebrate Pathology 89(1):78-84 DOI 10.1016/j.jip.2005.02.010.

Kalyaanamoorthy S, Minh BQ, Wong TKF, Von Haeseler A, Jermiin LS. 2017. ModelFinder: fast model selection for accurate phylogenetic estimates. Nature Methods 14(6):587-589 DOI 10.1038/nmeth.4285.

Kim MJ, Kim I. 2013. Description of nearly completed mitochondrial genome sequences of the garden chafer Polyphylla laticollis manchurica, endangered in Korea (insecta: coleoptera). International Journal of Industrial Entomology 27(1):185-202 DOI 10.7852/IJIE.2013.27.1.185.

Kim MJ, Im HH, Lee KY, Han YS, Kim I. 2014. Complete mitochondrial genome of the whiter-spotted flower chafer, protaetia brevitarsis (Coleoptera: Scarabaeidae). Mitochondrial DNA 25(3):177-178 DOI 10.3109/19401736.2013.792064.

Kim MJ, Jeong SY, Jeong J-C, Kim S-S, Kim I. 2016. Complete mitochondrial genome of the endangered flower chafer osmoderma opicum (Coleoptera: Scarabaeidae). Mitochondrial DNA Part B 1(1):148-149 DOI 10.1080/23802359.2016.1144104.

Kolmogorov M, Yuan J, Lin Y, Pevzner PA. 2019. Assembly of long, error-prone reads using repeat graphs. Nature Biotechnology 37(5):540-546 DOI 10.1038/s41587-019-0072-8.

Kumar S, Stecher G, Li M, Knyaz C, Tamura K. 2018. MEGA X: molecular evolutionary genetics analysis across computing platforms. Molecular Biology and Evolution 35(6):1547-1549 DOI 10.1093/molbev/msy096.

Li H. 2013. Aligning sequence reads, clone sequences and assembly contigs with BWA-MEM. arXiv. Available at http://arxiv.org/abs/1303.3997.

Li H. 2018. Minimap2: pairwise alignment for nucleotide sequences. Bioinformatics 34(18):3094-3100 DOI 10.1093/bioinformatics/bty191. 
Marshall SDG, Moore A, Vaqalo M, Noble A, Jackson TA. 2017. A new haplotype of the coconut rhinoceros beetle, Oryctes Rhinoceros, has escaped biological control by Oryctes Rhinoceros nudivirus and is invading Pacific Islands. Journal of Invertebrate Pathology 149:127-134 DOI 10.1016/j.jip.2017.07.006.

Mikheyev AS, Tin MMY. 2014. A first look at the oxford nanopore MinION sequencer. Molecular Ecology Resources 14(6):1097-1102 DOI 10.1111/1755-0998.12324.

Moore M, Sylla M, Goss L, Burugu MW, Sang R, Kamau LW, Kenya EU, Bosio C, Munoz Mde L, Sharakova M, Black WC, Kading RC. 2013. Dual African origins of global aedes aegypti s.l. populations revealed by mitochondrial DNA. PLOS Neglected Tropical Diseases 7(4):e2175 DOI 10.1371/journal.pntd.0002175.

Rambaut A. 2014. FigTree. version 1.4.2. Available at http:/tree.bio.ed.ac.uk/software/figtree/.

Regier JC, Shultz JW, Zwick A, Hussey A, Ball B, Wetzer R, Martin JW, Cunningham CW. 2010. Arthropod relationships revealed by phylogenomic analysis of nuclear protein-coding sequences. Nature 463(7284):1079-1083 DOI 10.1038/nature08742.

Reil JB, Jose MS, Rubinoff D. 2016. Low variation in nuclear and mitochondrial DNA inhibits resolution of invasion pathways across the Pacific for the coconut rhinoceros beetle (Scarabeidae: Oryctes Rhinoceros). Proceedings of the Hawaiian Entomological Society 48:57-69.

Rubinoff D, Holland BS, Shibata A, Messing RH, Wright MG. 2010. Rapid invasion despite lack of genetic variation in the erythrina gall wasp (Quadrastichus erythrinae Kim). Pacific Science 64(1):23-31 DOI 10.2984/64.1.023.

Sayadi A, Elina I, Christian T-R, Göran A. 2017. The evolution of dark matter in the mitogenome of seed beetles. Genome Biology and Evolution 9(10):2697-2706 DOI 10.1093/gbe/evx205.

Shelomi M, Lin S-S, Liu L-Y. 2019. Transcriptome and microbiome of coconut rhinoceros beetle (Oryctes Rhinoceros) larvae. BMC Genomics 20(1):957 DOI 10.1186/s12864-019-6352-3.

Shimodaira H, Hasegawa M. 1999. Multiple comparisons of log-likelihoods with applications to phylogenetic inference. Molecular Biology and Evolution 16(8):1114-1116

DOI 10.1093/oxfordjournals.molbev.a026201.

Song F, Li H, Shao R, Shi A, Bai X, Zheng X, Heiss E, Cai W. 2016. Rearrangement of mitochondrial tRNA genes in flat bugs (Hemiptera: Aradidae). Scientific Reports 6:25725 DOI 10.1038/srep25725.

Song N, Zhang H. 2018. The mitochondrial genomes of phytophagous scarab beetles and systematic implications. Journal of Insect Science 18(6):113 DOI 10.1093/jisesa/iey076.

Timmermans MJTN, Barton C, Haran J, Ahrens D, Culverwell CL, Ollikainen A, Dodsworth S, Foster PG, Bocak L, Vogler AP. 2016. Family-level sampling of mitochondrial genomes in coleoptera: compositional heterogeneity and phylogenetics. Genome Biology and Evolution 8(1):161-175 DOI 10.1093/gbe/evv241.

Trifinopoulos J, Nguyen L-T, Von Haeseler A, Minh BQ. 2016. W-IQ-TREE: a fast online phylogenetic tool for maximum likelihood analysis. Nucleic Acids Research 44(W1):W232-W235 DOI 10.1093/nar/gkw256.

Walker BJ, Abeel T, Shea T, Priest M, Abouelliel A, Sakthikumar S, Cuomo CA, Zeng Q, Wortman J, Young SK, Earl AM. 2014. Pilon: an integrated tool for comprehensive microbial variant detection and genome assembly improvement. PLOS ONE 9(11):e112963 DOI 10.1371/journal.pone.0112963.

Wang X-Y, Yang X-M, Lu B, Zhou L-H, Wu K-M. 2017. Genetic variation and phylogeographic structure of the cotton aphid, aphis gossypii, based on mitochondrial DNA and microsatellite markers. Scientific Reports 7(1):1920 DOI 10.1038/s41598-017-02105-4. 
Wick RR, Judd LM, Holt KE. 2019. Performance of neural network basecalling tools for oxford nanopore sequencing. Genome Biology 20(1):129 DOI 10.1186/s13059-019-1727-y.

Yang W, Zhang Y, Feng S, Liu L, Li Z. 2018. The first complete mitochondrial genome of the Japanese beetle Popillia japonica (Coleoptera: Scarabaeidae) and its phylogenetic implications for the superfamily Scarabaeoidea. Epub ahead of print 28 June 2018. International Journal of Biological Macromolecules 118(Pt B):1406-1413 DOI 10.1016/j.ijbiomac.2018.06.131.

Zhang D-X, Hewitt GM. 1997. Insect mitochondrial control region: a review of its structure, evolution and usefulness in evolutionary studies. Biochemical Systematics and Ecology 25(2):99-120 DOI 10.1016/S0305-1978(96)00042-7.

Zwick A. 2013. Degeneracy coding web service. PhyloTools. Available at http://www.phylotools. com/ptdegenwebservice.htm.

Zwick A, Regier JC, Zwickl DJ. 2012. Resolving discrepancy between nucleotides and amino acids in deep-level arthropod phylogenomics: differentiating serine codons in 21-amino-acid models. PLOS ONE 7(11):e47450 DOI 10.1371/journal.pone.0047450. 